\title{
Dynamic Biasing for Improved On-Orbit Total-Dose Lifetimes of Commercial Semiconductor Devices
}

\author{
Maximillian Holliday, Thomas A. Heuser, Zachary Manchester, Debbie G. Senesky
}

\begin{abstract}
The survivability of microelectronic devices in ionizing radiation environments drives spacecraft design, capability, mission-scope, and cost. This work exploits the periodic nature of many space radiation environments to extend device lifetimes without additional shielding or modifications to the semiconductor architecture. To improve component lifetimes via reduced total-dose accumulation, device bias was modulated during periods of intense irradiation. Simulation of this "dynamic biasing" technique applied to single-transistor devices in a typical low-Earth orbit results in an increase of component life from 5 days to 80 days (16x improvement) at the expense of $8 \%$ down time (92\% duty cycle). The biasing technique is also experimentally demonstrated using gamma radiation to study two device types spanning a range of integrated circuit complexity in $109 \mathrm{rad} / \mathrm{min}$ and $256 \mathrm{rad} / \mathrm{min}$ dose rate conditions. The significant improvements in device lifetimes demonstrated with the proposed dynamic biasing technique lays a foundation for more effective use of modern microelectronics for space applications. Analogous to the role real-time temperature monitoring plays in maximizing modern processor performance, the proposed dynamic biasing technique is a means of intelligently responding to the radiation environment and capable of becoming an integral tool in optimizing component lifetimes in space.
\end{abstract}

Index Terms-Charge carrier processes, consumer electronics, degradation, large scale integration, MOSFET, radiation effects, radiation inducted charge neutralization, total ionizing dose

\section{INTRODUCTION}

$\mathbf{T}$ HE bias condition of microelectronics during irradiation is known to play a significant role in the impact total ionizing dose (TID) has on device lifetime [1], [2]. However, aside from niche work investigating optimal device-specific bias states [3], [4], literature is lacking a fundamental study into using active device bias configuration as a means of mitigating device degradation due to TID, thereby extending device lifetimes in radiation environments.

The relationship between device bias and radiation-induced degradation is well-studied, and can be briefly summarized as an electrostatic potential across a dielectric region causing less recombination of radiation-induced electron/hole $(\mathrm{e} / \mathrm{h})$ pairs [5]. The electrostatic forces combined with vast differences in oxide carrier mobilities results in an increase of trapped charges that is directly dependent on the electric field, oxide type, and oxide thickness [6]. It is an accumulation of these trapped charges inside microelectronics that causes the gradual

M. H. and T.H. Authors are with the Department of Materials Science and Engineering, Stanford University, Stanford, California 94305, USA.

Z. M. Author was with Stanford University, Stanford, California 94305, USA. He is now with the Robotics Institute, Carnegie Mellon University, Pittsburgh, PA 15213 USA.

D. S. Author is with Department of Aeronautics and Astronautics, Stanford University, Stanford, California 94305, USA. degradation of device performance as a function of TID, ultimately resulting in permanent failure of the device.

For field-effect transistors, TID degradation commonly manifests as positive or negative shifts in threshold voltage $\left(\Delta V_{T H}\right)$ as a result of charge trapping [7]. The $\Delta V_{T H}$ formed by radiation-induced trapped charges has many similarities to traditional metal-oxide-semiconductor (MOS) aging mechanisms such as hot-electron-induced (HIT) and positive/negative bias temperature instability (PBTI, NBTI) [8], [9]. In both cases, sensitive oxide regions, such as those located at the gate, isolation, and below the substrate are the primary contributors to the $\Delta V_{T H}$. However, the regions of oxide vulnerable to charge trapping and the rate at which charge accumulation occurs helps distinguish radiationinduced degradation from those of physical aging.

In the case of TID degradation, the magnitude of $\Delta V_{T H}$ can be estimated by the accumulated dose inside sensitive oxide regions [2]. Beginning with a simple approximation of MOSFET device behavior, the threshold voltage $\left(V_{T H}\right)$ can be described as proportional to the charge of the gate oxide $\left(Q_{o x}\right)$, and inversely proportional to gate oxide capacitance $\left(C_{o x}\right)$. This relationship between $V_{T H}$ and oxide charge allows for an estimation of $\Delta V_{T H}$ as a function of radiation dose as described by Krantz [10]

$$
\Delta V_{T H}=V_{T H}^{0}-\frac{1}{C_{o x}} \int_{0}^{t_{o x}} f\left(E_{o x}\right) D g_{0} t_{o x}\left(1-\frac{x}{t_{o x}}\right) d x
$$

where $V_{T H}^{0}$ is the threshold voltage before irradiation, $t_{o x}$ is the thickness of the gate oxide, $f\left(E_{o x}\right)$ is the hole yield, $D$ is the dose (rad) deposited into the material, $g_{0}$ is the e/h pair per $\mathrm{rad}$ (pairs $/ \mathrm{cm}^{3}$ ), and $\mathrm{x}$ is the distance in to the oxide. Device bias influences the hole yield term in (1) according to the relationship

$$
f\left(E_{\text {ox }}\right)=\left(1+\frac{0.55}{|E|}\right)^{-0.7}
$$

where $E$ is the electric field in $\mathrm{MV} / \mathrm{cm}$ across the gate dielectric. Equation (2) describing hole yield has been empirically determined and is limited to describing the fraction of unrecombined holes persisting in $\mathrm{SiO}_{2}$ from $\mathrm{Co}-60$ generated gamma exposure [11]. Therefore, the hole yield is dependent on the dielectric material, ionizing radiation type, and the magnitude of electric field present in the material.

The usefulness of dynamically changing device bias to extend component lifetimes is well illustrated in the context of Earth's orbital radiation environment. To aid in visualizing 

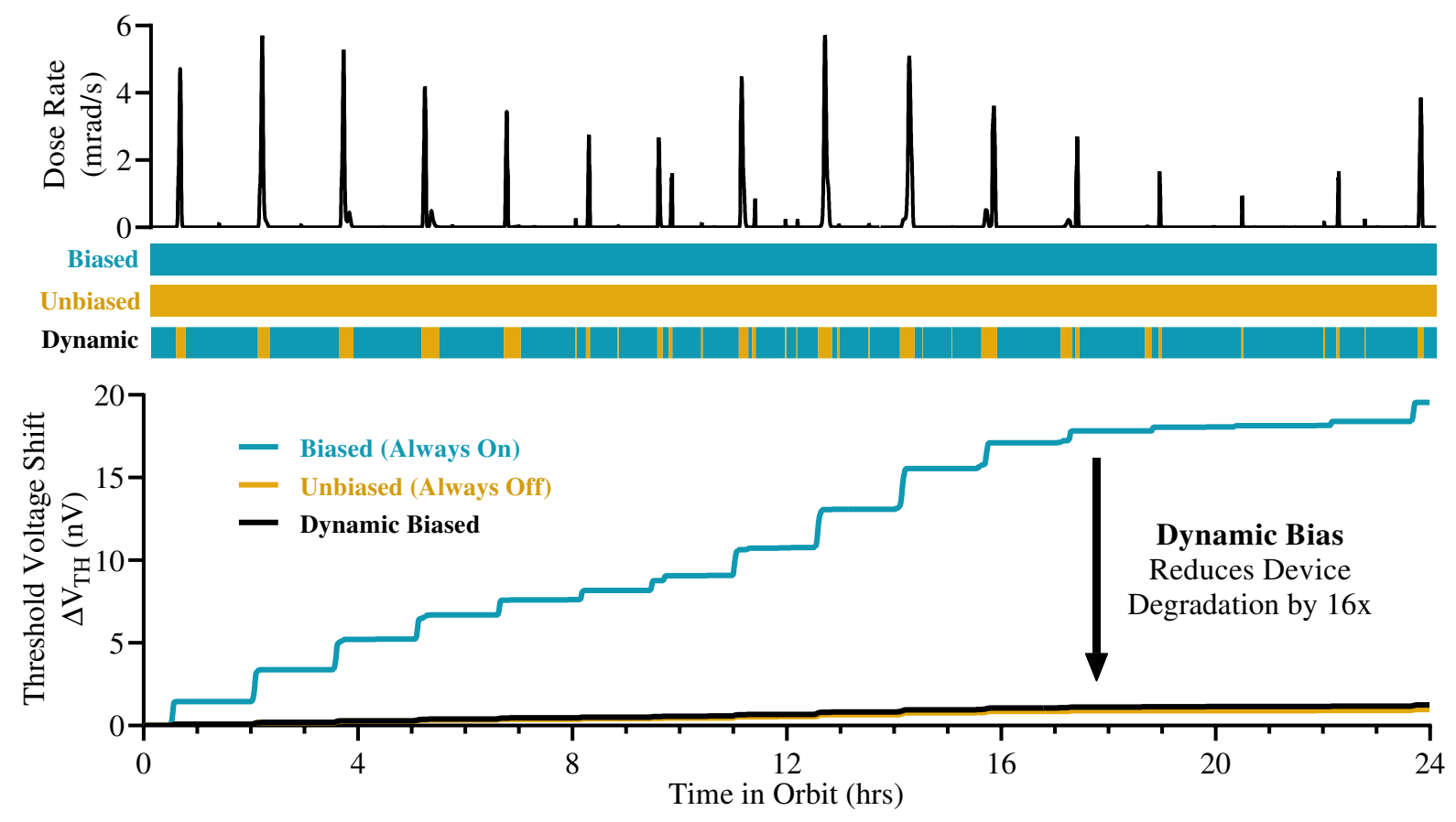

Fig. 1: Simulated $24 \mathrm{hr}$ on-orbit dose rate time series (top) illustrating periodic radiation environment and resulting $\Delta V_{T H}$ (bottom) for a $0.5 \mathrm{~mm} \mathrm{Al-shielded} \mathrm{P-channel} \mathrm{MOSFET} \mathrm{biased} \mathrm{continuously} \mathrm{(bottom,} \mathrm{blue,} V_{D S}=-3.3 \mathrm{~V}$ ), never (bottom, yellow, $V_{D S}=0 \mathrm{~V}$ ), and dynamically (bottom, black, $V_{D S}=0$ to $-3.3 \mathrm{~V}$ ). Biasing state as a function of orbit time is illustrated for for all three conditions (middle) resulting in a 16x reduction in $\Delta V_{T H}$, thereby improving the TID lifetime of the simulated device by $16 x$ at the expense of $8 \%$ down time (92\% duty cycle).

the periodic nature of ionizing dose accumulation in satellites, the total dose inside a simple spacecraft occupying a common low-Earth orbit (LEO) has been modeled in Fig. 1. As shown, a typical LEO satellite orbiting at an altitude similar to the International Space Station $(408 \mathrm{~km})$ will experience brief but intense moments of high dose rate irradiation that are separated by longer periods of little-to-no trapped-particle activity. These transient high dose rate events occur on intervals equal to a typical orbital period for LEO objects (about 90 minutes) and frequently last for minutes, making the transient radiation environment ideal for potential dynamic biasing benefits.

This work studied a "dynamic biasing" scheme for devices as a means of controlling radiation-induced carrier lifetimes and the resulting impact on device degradation for commercial field-effect devices. Simulations using (1) and (2) were integrated into modern trapped-particle models to generate $\Delta V_{T H}$ predictions, then experimentally verified for two commercial devices that span a wide range of integrated circuit complexity. In-situ measurements collected during radiation testing are presented for a variety of bias conditions to illustrate the effectiveness of the proposed dynamic biasing technique at mitigating TID effects.

\section{EXPERIMENTAL DETAILS}

\section{A. On-Orbit Simulation Parameters}

The proposed dynamic biasing technique was modeled for a simple metal oxide field effect transistor geometry in a typical
low-Earth orbit. The simulated radiation environment was constructed using AE9, AP9 models for specifying the trapped energetic particle and space plasma environment [12]. The simulated orbit was propagated using the Kepler J2 model for a nominal $408 \mathrm{~km}$ orbit using the following orbital elements: an inclination of $97.06^{\circ}$, a right ascension of the ascending node of $130.53^{\circ}$, an apogee and perigee altitude of $480 \mathrm{~km}$, and an argument of latitude of $180^{\circ}$ for a one year duration occurring from January 1, 2015 to January 1, 2016. The SHIELDOSE$2 \mathrm{Q}$ model was then used to calculate dose rates from the simulated orbit for an $\mathrm{SiO}_{2}$ point detector centered inside a solid aluminum sphere of radius $0.5 \mathrm{~mm}$ [13]. Resulting dose rates were integrated on five second intervals to produce a time series of total dose data for the simulated mission. Equation (1) was used to calculate an estimated $\Delta V_{T H}$ for a simple P-channel MOSFET with a $100 \mathrm{~nm}$ thick $\mathrm{SiO}_{2}$ gate oxide for the simulated total dose values at various bias conditions.

TABLE I: Experimentally Tested Commercial Devices

\begin{tabular}{cccc}
\hline $\begin{array}{c}\text { Part } \\
\text { Number }\end{array}$ & Description & $\begin{array}{c}\text { Architecture, } \\
\text { \# Transistors }\end{array}$ & $\begin{array}{c}\text { Performance } \\
\text { Metric }\end{array}$ \\
\hline $\begin{array}{c}\text { IRLML2803 } \\
\text { (Infineon) }\end{array}$ & $\begin{array}{c}\text { MOSFET } \\
\text { (N-Channel) }\end{array}$ & $\begin{array}{c}\text { "HexFET" } \\
1 \text { Transistor }\end{array}$ & $\Delta V_{T H}$ \\
\hline $\begin{array}{c}\text { IRLML5103 } \\
\text { (Infineon) }\end{array}$ & $\begin{array}{c}\text { MOSFET } \\
\text { (P-Channel) }\end{array}$ & $\begin{array}{c}\text { "HexFET" } \\
1 \text { Transistor }\end{array}$ & $\Delta V_{T H}$ \\
\hline $\begin{array}{c}\text { TPS82740 } \\
\text { (TI) }\end{array}$ & $\begin{array}{c}\text { Switching } \\
\text { Regulator }\end{array}$ & $\begin{array}{c}\text { SOI } \\
\text { 1000 Transistors }\end{array}$ & $\begin{array}{c}\text { Output } \\
\text { Voltage }\end{array}$ \\
\hline
\end{tabular}




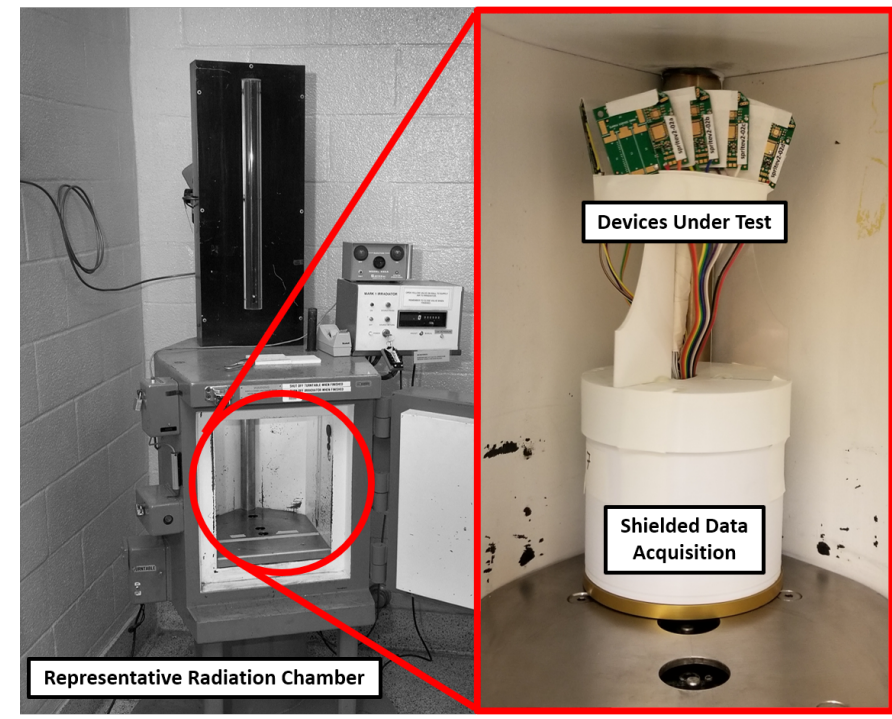

Fig. 2: Representative J.L. Shepherd and Associates Mark I gamma radiation chamber (left, [15], [16] and in-situ test fixture (right) with $\mathrm{Pb} / \mathrm{Al}$ shielded data acquisition enclosure supporting the devices under test.

\section{B. Device Selection}

Device functionality as a function of bias condition during irradiation was studied across two levels of commercial VLSI integration complexity. Table 1 outlines each device category with relevant fabrication technology used, the approximate number of transistors in the device, and the performance metric used when reporting radiation tolerance.

General device architecture, fabrication details, and estimated transistor counts were collected using visible-light spectroscopy to examine representative samples from each device group. Spectroscopy samples were prepared via chemical decapsulation following common failure analysis techniques [14]. Devices were submerged in $96 \%$ sulfuric acid at $150^{\circ} \mathrm{C}$ for 20 minutes, then rinsed with acetone and again with deionized water before collecting micrographs on an Olympus stereo microscope.

\section{Radiation Test Environment and Setup}

TID testing was performed under constant gamma irradiation conditions to achieve total dose accumulations that meet or exceed TID values simple spacecraft might experience after a multi-year mission in LEO. Testing was conducted following MIL-STD-883 Method 1019.8 with the exception of a Cs137 source used in place of Co-60 [17]. A J.L. Shepherd and Associates Mark I model gamma radiation chamber similar to the instrument shown in Fig. 2 from Brookhaven National Laboratory was used at the configurable dose rates of 109 and $256 \mathrm{rad} / \mathrm{min}$ [15], [16]. Solid-state dosimeters from Tyndall National Institute (Part Number: TD1003, $200 \mu \mathrm{m}$ "radFETs") were used for in-situ dose characterization.

Separate electrical measurements were necessary to monitor the performance metrics of each device during radiation exposure. Aside from the devices under test, all data logging equipment was housed inside the shielded enclosure shown

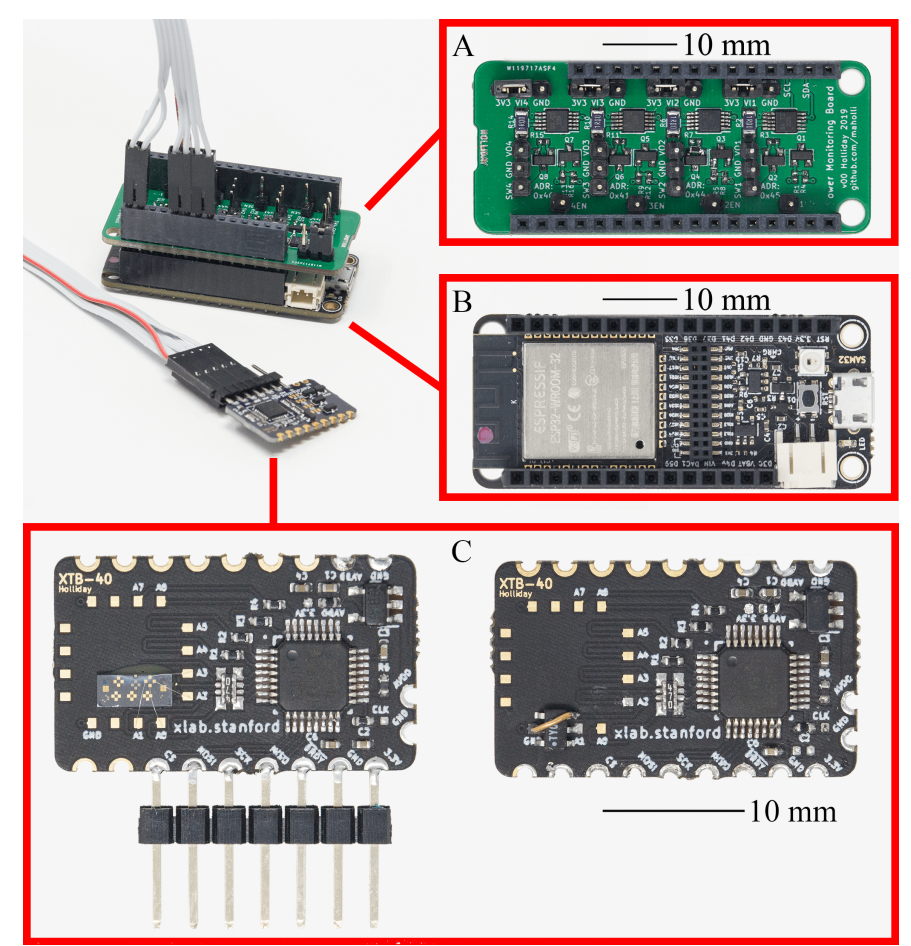

Fig. 3: Modular in-situ data acquisition hardware setup used to operate and monitor device samples during irradiation featuring (A) power monitoring and MOSFET switching array module, (B) primary computing and data storage module, and (C) two instances of a versitle 28-bit ADC module driving and measuring custom wirebonded devices (left) and a MOSFET dosimeter (right).

in Fig. 2 and constructed of layered aluminum, lead, and polyethylene terephthalate glycol (PETG) with thicknesses of $1.6 \mathrm{~mm}, 6.4 \mathrm{~mm}$, and $2 \mathrm{~mm}$, respectively. Attenuation performance of the shielded enclosure was monitored using a TD1003 radFET located in the center of the shielded volume which verified the cumulative dose remained below $100 \mathrm{rad}$ throughout the duration of each experiment.

Figure 3 depicts the open-source data acquisition hardware developed and used to conduct electrical measurements, insitu, during irradiation. The modular hardware design enabled operation and monitoring of the simple transistor devices and dosimeters as well as the highly integrated switching regulators while maintaining a form factor suitable for the shielded enclosure. Shown in Fig. 3 is the primary computing module (middle) utilizing a 32-bit ARM microcontroller (Part Number: Microchip ATSAMD51J20A) with on-board microSD socket and external power management, as well a mezzanine board (top) containing an array of four MOSFET switches and power monitoring capability. Illustrated at the bottom of Fig. 3, is a carrier board capable of housing bare die wirebonded to pads (not used in this study) as well as common surface mount packages (SOT-23, SOT-363) while utilizing a versatile 28-bit analog-to-digital converter (Part Number: TI ADS124S08) to drive devices under test via the on-chip programmable current sources, voltage references, temperature sensor, and general purpose I/O. Power 

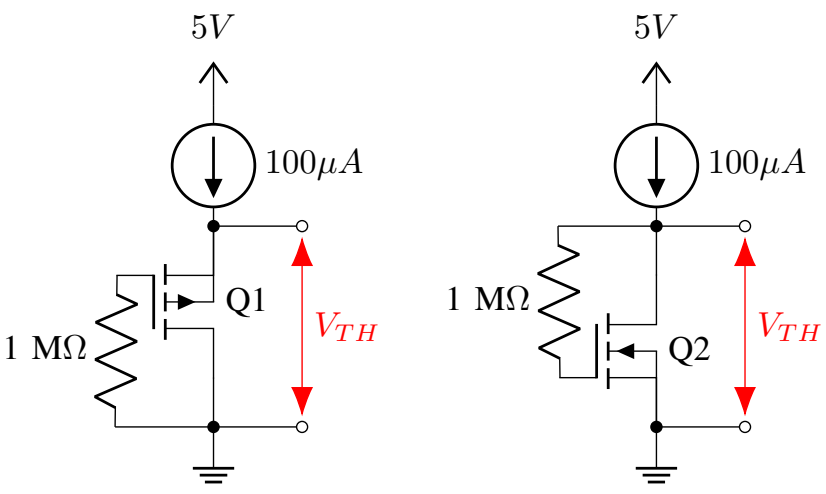

Fig. 4: Direct $V_{T H}$ readout circuit for commercial P-Channel (left) and N-Channel (right) MOSFET devices using an external current source.

to the acquisition hardware was supplied from lithium ion cells (Part Number: LG INR1860 MJ1 3500 mAh) housed inside the shielded enclosure. Schematics, design files, and manufacturing materials are available on the author's GitHub for the processing module: https://github.com/maholli/SAM32, and ADC module: https://github.com/maholli/XTB.

\section{Device Operation and $V_{T H}$ Measurement Technique}

Threshold voltage was monitored during irradiation for commercial single-transistor P-channel and N-channel devices using the direct $V_{T H}$ readout circuit shown in Fig. 4. The $V_{T H}$ readout method used is similar to the method described by Andjelković et al. for using MOSFETs as dosimeters [18]. Rather than using Andjelković's method of driving current into the bulk of the device, which is not always feasible with commercially packaged devices, Fig. 4 illustrates our adaptation that allows current to be driven into the source (internally shorted to the bulk) for PMOS devices, or the drain for NMOS devices. Additionally, our adaptation includes a 1 $M \Omega$ resistor connected between the gate contact and ground (PMOS) or the gate contact and drain (NMOS) in order to achieve proper $V_{G S}$ bias with limited gate leakage. Principal of operation for the simple circuits shown in Fig. 4 as direct $V_{T H}$ measurement tools relies on the inherent nature of the current source to continuously adjust the voltage necessary to drive the desired current. As configured, the supplied current must flow through the channel of the MOSFET which is only possible once the device's $V_{T H}$ has been met and the transistor turns on. For the circuits shown in Fig. 4, $V_{T H}$ is met as soon as the current source reaches a voltage adequate to achieve the $100 \mu \mathrm{A}$ output, thereby turning on the transistor Q1 or Q2 and also providing a convenient location for direct measurement of the the potential (shown in red). Using this technique, biased MOSFET samples were continuously driven at constant current throughout irradiation with voltage measurements performed at a frequency of $0.1 \mathrm{~Hz}$. Unbiased MOSFET samples had source and drain contacts grounded during irradiation aside from a 0.5 second configuration and measurement routine performed at a frequency of $0.1 \mathrm{~Hz}$. In both sample groups, the current source within the $V_{T H}$ readout circuit was configured to drive $100 \mu \mathrm{A}$ during measurement collection.

The performance of the heavily-integrated TPS 82740 switching regulator was quantified by measuring the voltage output under a constant load of $10 \mathrm{~mA}$ during irradiation. The regulated output voltage is a result of the integrated step down converter circuit comprised of thousands of transistors providing device features such as control logic, comparators, and voltage references. The devices under test were configured to output $2.1 \mathrm{~V}$ and an array of shielded MOSFET switches were used to control regulator input supply according to the relevant duty cycle $(75 \%, 50 \%, 25 \%)$ with a period of 0.016 $\mathrm{Hz}(60 \mathrm{sec})$.

\section{RESUlTS AND DISCUSSION}

\section{A. On-Orbit Simulation Results}

Simulated dose rates as a function of orbit time are shown in Fig. 1 (bottom) for a 24 hour period. The transient dose rate spikes are not an artifact of the particle models; rather, the sudden rise in dose rate is caused by localized regions of trapped electrons and protons present in Earth's orbit [19]. The simulated orbit encounters dose rate spikes on 90-minute intervals that typically last less than 10 minutes. The resulting $\Delta V_{T H}$ for the simulated P-channel device are illustrated in Fig. 1 (top) for three bias conditions: always biased (blue), never biased (yellow), and dynamically biased (black). The total $\Delta V_{T H}$ for the 24 hour period are $19.55 \mathrm{mV}, 0.98 \mathrm{mV}$, and $1.24 \mathrm{mV}$, respectively. The simulated dynamically biased case was powered off for dose rates exceeding $0.001 \mathrm{mrad} / \mathrm{sec}$, resulting in a $16 \mathrm{x}$ reduction in $\Delta V_{T H}$ as compared to the always-biased case, at the expense of an $8 \%$ reduction in duty cycle (92\% time on) for the 24 hour period.

Consider the on-orbit simulation findings applied to a circuit containing the modeled P-channel device, exposed to the simulated on-orbit environment, and able to maintain nominal operation as long as the respective PMOS device maintains a $V_{T H}$ tolerance of $10 \%$. Given this scenario, the simulated $\Delta V_{T H}$ predicts circuit failure after 5.1 days for an alwaysbiased PMOS device, whereas the dynamically biased device would extend circuit lifetime to 80.5 days (a 16x, or $1600 \%$ improvement).

The dose rate cutoff threshold, chosen as $0.001 \mathrm{mrad} / \mathrm{sec}$ for the above simulation, is a powerful means of tuning the

TABLE II: Summary of Experimentally Determined MOSFET and Regulator Radiation Performance

\begin{tabular}{ccccc}
\hline Description & $\begin{array}{c}\text { Part } \\
\text { Number }\end{array}$ & $\begin{array}{c}\text { Duty } \\
\text { Cycle }\end{array}$ & $\begin{array}{c}\text { Functional } \\
\text { Dose Limit }\end{array}$ & $\begin{array}{c}\text { Lifetime } \\
\text { Improvement }\end{array}$ \\
\hline \multirow{2}{*}{$\begin{array}{c}\text { Switching } \\
\text { Regulator }\end{array}$} & TPS82740 & $25 \%$ & $22 \mathrm{krad}$ & \\
& TPS82740 & $50 \%$ & $22 \mathrm{krad}$ & $57 \%$ \\
TPS82740 & $75 \%$ & $14 \mathrm{krad}$ & \\
\hline $\begin{array}{c}\text { N-Channel } \\
\text { MOSFET }\end{array}$ & IRLML2803 & $0 \%$ & $34 \mathrm{krad}$ & $13 \%$ \\
\hline IRLML2803 & $100 \%$ & $30 \mathrm{krad}$ & \\
MOSFET & IRLML5103 & $0 \%$ & $39 \mathrm{krad}$ & $44 \%$ \\
\hline
\end{tabular}




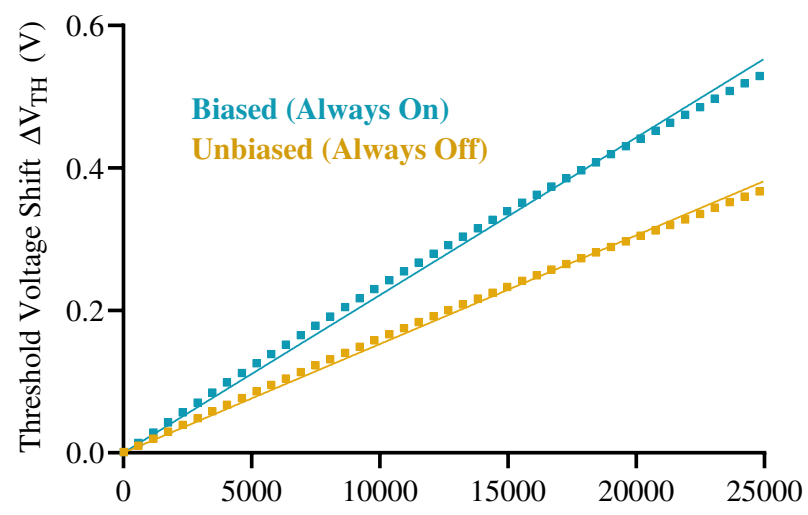

(a)

Dose $(\mathrm{rad})$

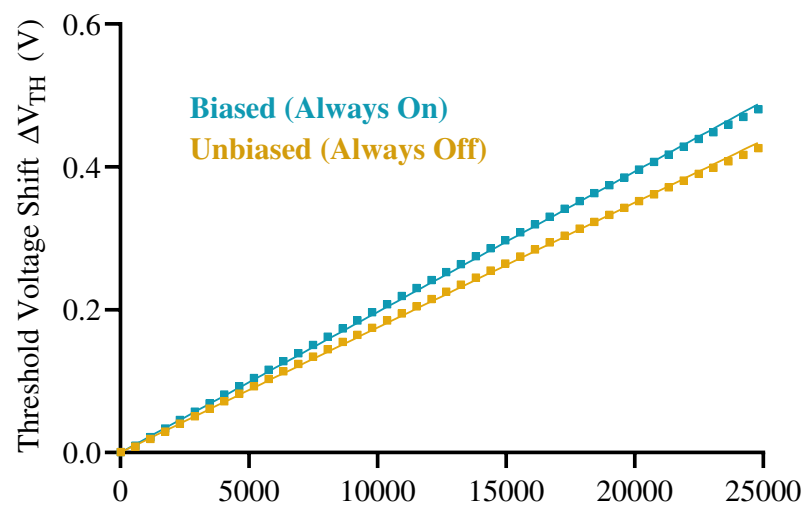

(b)

Dose (rad)

Fig. 5: Experimentally measured $V_{T H}$ shifts for biased (blue, $V_{D S}= \pm 3.3 \mathrm{~V}$ ) and unbiased (yellow, $V_{D S}=0 \mathrm{~V}$ ) commercial P-channel (a) and N-channel (b) power MOSFETS exposed to gamma radiation at a rate of $256 \mathrm{rad} / \mathrm{min}$ to $25 \mathrm{krad}$.

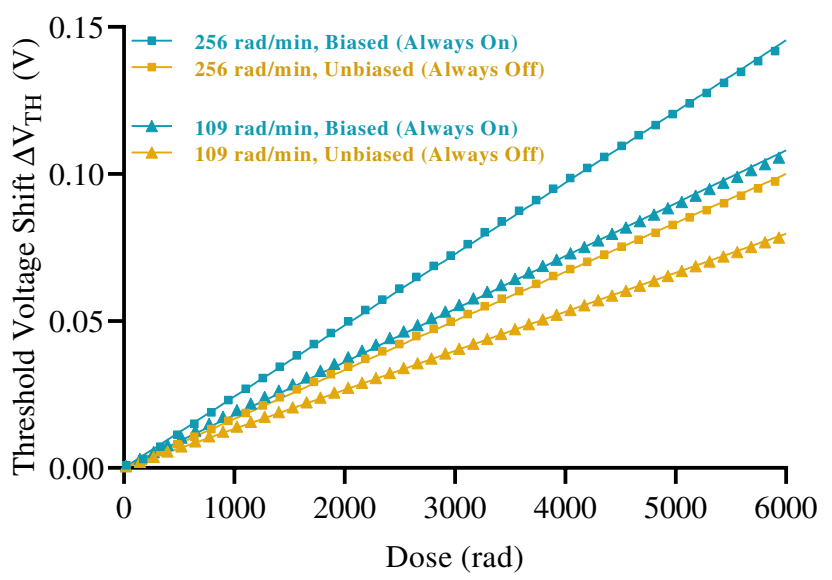

Fig. 6: Experimentally measured $V_{T H}$ shifts for biased (blue, $V_{D S}= \pm 3.3 \mathrm{~V}$ ) and unbiased (yellow, $V_{D S}=0 \mathrm{~V}$ ) commercial P-channel MOSFETs irradiated at $256 \mathrm{rad} / \mathrm{min}$ (squares) and $109 \mathrm{rad} / \mathrm{min}$ (triangles) to a total dose of $6 \mathrm{krad}$.

dynamically biased electronic system to balance radiationinduced degradation with the needs of the mission. For example, consider extending the operational lifetime of a satellite by adjusting these cutoff thresholds at the system or even subsystem level to achieve the desired effective dose for components or subsystems with known lifetime limiting TID limits.

\section{B. Experimental Results: MOSFETs}

Figure 5 summarizes the in-situ $V_{T H}$ measurements collected for biased and unbiased IRLML2803 and IRLML5104 devices as a function of total dose. A line through $(\mathrm{X} 0, \mathrm{Y} 0)$ following a least-squares fit is shown for each sample as a means of quantifying the differential $V_{T H}$ (units of $\frac{\Delta V_{T H}}{\operatorname{rad}}$ ). Samples exposed at a rate of $256 \mathrm{rad} / \mathrm{min}$ to a total dose of 25 krad exhibit differential $V_{T H}$ of $2.213 \times 10^{-5} \frac{\Delta V_{T H}}{\text { rad }}$ for PMOS and $1.967 \times 10^{-5} \frac{\Delta V_{T H}}{r a d}$ for NMOS if biased continuously during irradiation, whereas the differential $V_{T H}$ for unbiased devices were found to be $1.526 \times 10^{-5} \frac{\Delta V_{T H}}{\mathrm{rad}}$ for PMOS and $1.749 \times 10^{-5} \frac{\Delta V_{T H}}{r a d}$ for NMOS. The functional dose limits are summarized in Table II and estimated using the differential $V_{T H}$ to determine the dose necessary to exceed a $\Delta V_{T H}$ of $0.6 \mathrm{~V}$ (a tolerance of $\pm 33 \%$ shift in the specified $V_{T H}$ of 1.8 $\mathrm{V})$ for each respective device.

As expected, device bias during constant irradiation has a significant impact on the threshold voltage for simple singletransistor devices such as the IRLML2803 and IRLML5103 MOSFETs. In-situ monitoring of the MOSFET devices shows PMOS devices failing "off" and NMOS failing "on," which is congruent with the simple device physics model discussed earlier. The significant difference in estimated lifetime improvements between NMOS and PMOS are to be expected. As discussed earlier, gamma-induced TID degradation in $\mathrm{SiO}_{2}$ can be quantified as the number of uncombined holes persisting in relevant oxide layers such as gate and isolation regions. Recalling that NMOS devices rely on electrons as the primary charge-carrier, these devices have inherently more opportunities for e/h recombination, especially during operation, than their PMOS counterparts [7]. The result of increased e/h recombination manifests as smaller lifetime improvements for dynamically biased NMOS devices as compared to PMOS. Interface trap concentrations and device architecture are two parameters that directly influence this behavior.

The impact of dose rate was studied in more detail for PMOS by irradiating sets of IRLML2803 devices to $6 \mathrm{krad}$, as illustrated in Fig. 6, at a rate of 256 (blue) and 109 (gray) $\mathrm{rad} / \mathrm{min}$. The slower dose rate samples exhibit lower differential $V_{T H}$ values: $1.800 \times 10^{-5}$ for biased, and $1.328 \times 10^{-5}$ for unbiased, whereas the faster dose rate samples are consistent with behavior shown previously: $2.424 \times 10^{-5}$ biased, and $1.667 \times 10^{-5}$ unbiased. The observed differential $V_{T H}$ dependency on dose rate is believed to be the result of 


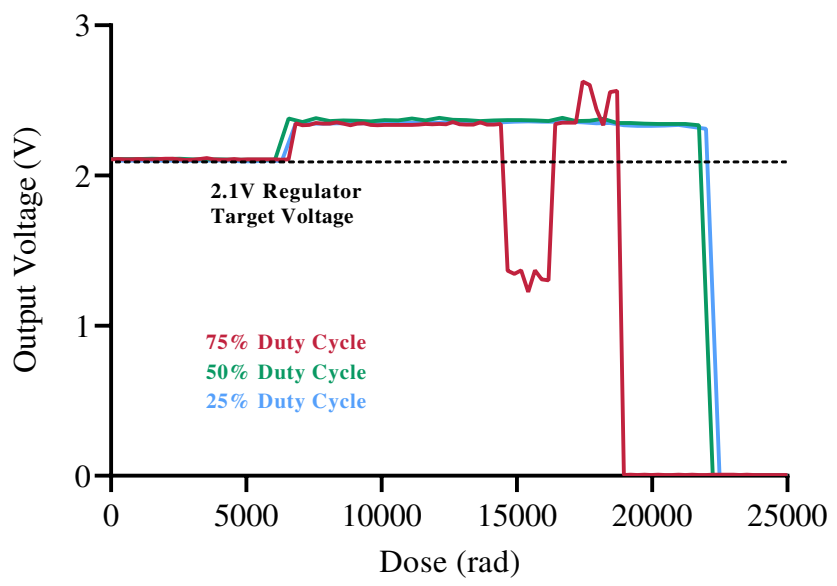

Fig. 7: Output voltage measured in-situ during irradiation for commercial TPS82740 switching regulators under constant 10 $\mathrm{mA}$ load as function of dose for red: $75 \%$, green: $50 \%$, and blue: $25 \%$ duty cycled devices.

inherent thermal recombination of e/h pairs as described by Oldham [20]. Given the constant exposure environment used for this dynamic biasing work, the $44 \%$ lifetime improvement for PMOS and 13\% improvement for NMOS are expected to increase in periodic radiation environments such as LEO. These dose rate findings should be an important parameter for future real-time implementations of the dynamic biasing technique.

Extrapolating the linear relationship between threshold voltage and dose for the $109 \mathrm{rad} / \mathrm{min}$ rate illustrated in Fig. 6, it can be estimated that the IRLML2803 will no longer meet the specified $V_{T H}$ of $1.8 \mathrm{~V} \pm 33 \%$ at an approximate total dose of $33 \mathrm{krad}$ (biased) and $45 \mathrm{krad}$ (unbiased). This is in strong agreement with TID performance reported by O'Bryan [21] of $35 \mathrm{krad}$ for the IRLML2803.

\section{Experimental Results: Switching Regulators}

Figure 7 illustrates the output voltage performance of the TPS82740 switching regulator under constant $10 \mathrm{~mA}$ load as measured during irradiation at a rate of $256 \mathrm{rad} / \mathrm{min}$. The complex output behavior observed in Fig. 7 as a result of the irradiation is broken down into three regions and interpreted below.

1) The first observed event is a jump in output voltage from $2.1 \mathrm{~V}$ to $2.3 \mathrm{~V}$ seen at about $6.5 \mathrm{krad}$ for all devices. The TPS82740 regulators use an internal feedback divider network to configure the output voltage to be one of eight possible values: $1.8 \mathrm{~V}$ to $2.6 \mathrm{~V}$ in $0.1 \mathrm{~V}$ increments. Therefore, the observed jump in output voltage is believed to be a result of a $V_{T H}$ shift in the error amplifier and/or main comparator of the feedback loop to yield a new set-point to the control logic. Possible issues with the data acquisition circuit were ruled out after confirming all internal health checks were valid for the duration for the experiment and verifying the new output voltage was an intentional feature of the device. The change in output voltage at $6.5 \mathrm{krad}$ was not considered device failure since it is within the operating parameters of the device.

2) The second event is a sudden drop-out and subsequent recovery (with overshoot) of output voltage beginning at $14 \mathrm{krad}$ for the $75 \%$ duty cycled device. Although there is partial recovery, the drop in output voltage at 14 krad was considered to be device failure for this sample. One possible explanation for the interesting recovery behavior after about $2.4 \mathrm{krad}$ (9.3 minutes at the dose rate of $256 \mathrm{rad} / \mathrm{min}$ ) is a result of the $1.7 \mathrm{MHz}$ switching frequency of the regulator design. Rapidly changing bias conditions have been shown to improve recombination rates [22]. At the $14 \mathrm{krad}$ point of failure, once the output voltage has dropped to $1.3 \mathrm{~V}$ it is feasible a localized net-gain of e/h recombination could form as a result of the lower bias. The annihilation of these TID induced trapped charges is one method for the comparator to recover after a period of time.

3) Finally, the staggered and abrupt failure mode of all three devices is observed from $19 \mathrm{krad}$ to $22 \mathrm{krad}$. The sudden and precise drop in output voltage from $2.3 \mathrm{~V}$ to $0 \mathrm{~V}$ suggests this failure mode to be a result of triggering one or more of the protection features present in TPS82740 regulator design. For example, radiation induced $V_{T H}$ shifts in the input control and comparator subsystems could readily engage the TPS82740's undervoltage lockout or logic-controlled enable/disable feature, resulting in the observed voltage cutoff behavior. Separate testing of each functional block comprising the TPS82740 regulator would be necessary, and outside the scope of this work, to conclude root cause of the observed regulator behavior.

Intermittent failures, as seen in the $75 \%$ duty cycle case, and non-linear component lifetimes as function of TID are expected for highly integrated devices with many subsystems such as the TPS 82740 regulator. However, with a better understanding of the complex in-situ behavior during irradiation, it's clear that bias condition continues to impact overall component lifetime of the heavily integrated switching regulator resulting in an observed $57 \%$ improvement for the $50 \%$ duty cycle case as compared to the $75 \%$ case.

\section{CONCLUSIONS AND Future WORK}

This work establishes the feasibility of using the proposed "dynamic biasing" technique as a means of improving TID lifetimes for commercially available microelectronic devices with single-transistor and $>1000$ transistor complexities. Trapped particle models were used to simulate a 16x improvement in on-orbit transistor lifetimes using the proposed biasing technique. Simulated results were demonstrated experimentally via in-situ device monitoring during gamma irradiation for two levels of commercial IC complexity. The biasing technique is found to improve single-transistor commercial device lifetimes by $44 \%$ for PMOS and $13 \%$ for NMOS, whereas the lifetime for a heavily integrated switching regulator was improved by $57 \%$. 
Experimental results show dose rate continues to play a role in lifetimes of dynamically biased PMOS and NMOS devices. Continued research is planned to study the effect of dynamic biasing at the multi-component system level, including methods of integrating responsive sensor systems to provide real-time and intelligent response in volatile radiation environments.

\section{ACKNOWLEDGMENT}

The authors would like to thank Sharmila Bhattacharya and Sergio R. Santa Maria from NASA Ames Research Center, Moffett Field, California, for access to their radiation facilities.

\section{REFERENCES}

[1] James R. Schwank, "Basic Mechanisms of Radiation Effects in the Natural Space Radiation Environment," Tech. Rep., 1994.

[2] D. M. Fleetwood, "Total Ionizing Dose Effects in MOS and Low-DoseRate-Sensitive Linear-Bipolar Devices," IEEE Transactions on Nuclear Science, vol. 60, no. 3, pp. 1706-1730, Jun. 2013.

[3] F. Smith and S. Mostert, "Total Ionizing Dose Mitigation by Means of Reconfigurable FPGA Computing," Ph.D. dissertation, University of Stellenbosch, Aug. 2007.

[4] C. J. Gonzalez Aguilera, R. G. Vaz, M. B. Oliveira, V. W. Leorato, O. L. Goncalez, and T. R. Balen, "TID Effects on a Data Acquisition System With Design Diversity Redundancy," IEEE Transactions on Nuclear Science, vol. 65, no. 1, pp. 583-590, Jan. 2018.

[5] H. J. Barnaby, "Total-Ionizing-Dose Effects in Modern CMOS Technologies," IEEE Transactions on Nuclear Science, vol. 53, no. 6, pp. 3103-3121, Dec. 2006.

[6] V. V. Orlov, V. A. Felitsyn, and G. I. Zebrev, "Compact modeling of I-V characteristics in irradiated MOSFETs: Impact of operation temperature and interface traps," in 2016 16th European Conference on Radiation and Its Effects on Components and Systems (RADECS). Bremen: IEEE, Sep. 2016, pp. 1-4.

[7] D. M. Fleetwood, P. S. Winokur, R. A. Reber, T. L. Meisenheimer, J. R. Schwank, M. R. Shaneyfelt, and L. C. Riewe, "Effects of oxide traps, interface traps, and "border traps" on metal-oxide-semiconductor devices," Journal of Applied Physics, vol. 73, no. 10, pp. 5058-5074, May 1993.

[8] Chenming Hu, Simon C. Tam, Fu-Chieh Hsu, Ping-Keung Ko, Tung-Yi Chan, and K. W. Terrill, "Hot-Electron-Induced MOSFET Degradation - Model, Monitor, and Improvement," IEEE Journal of Solid-State Circuits, vol. 20, no. 1, pp. 295-305, Feb. 1985.

[9] V. Huard, C. R. Parthasarathy, C. Guerin, and M. Denais, "Physical Modeling of Negative Bias Temperature Instabilities for Predictive Extrapolation," in 2006 IEEE International Reliability Physics Symposium Proceedings, Mar. 2006, pp. 733-734.

[10] R. J. Krantz, L. W. Aukerman, and T. C. Zietlow, "Applied Field and Total Dose Dependence of Trapped Charge Buildup in MOS Devices," IEEE Transactions on Nuclear Science, vol. 34, no. 6, pp. 1196-1201, Dec. 1987.

[11] M. Shaneyfelt, D. Fleetwood, J. Schwank, and K. Hughes, "Charge yield for cobalt-60 and 10-keV X-ray irradiations of MOS devices," IEEE Transactions on Nuclear Science, vol. 38, no. 6, pp. 1187-1194, Dec./1991.

[12] G. P. Ginet, T. P. O'Brien, S. L. Huston, W. R. Johnston, T. B. Guild, R. Friedel, C. D. Lindstrom, C. J. Roth, P. Whelan, R. A. Quinn, D. Madden, S. Morley, and Y.-J. Su, "AE9, AP9 and SPM: New Models for Specifying the Trapped Energetic Particle and Space Plasma Environment," Space Science Reviews, vol. 179, no. 1-4, pp. 579-615, Nov. 2013.

[13] S. M. Seltzer, "Electron, Electron-Bremsstrahlung and Proton DepthDose Data for Space-Shielding Applications," IEEE Transactions on Nuclear Science, vol. 26, no. 6, pp. 4896-4904, 1979.

[14] M. Jacques, "The Chemistry of Failure Analysis," in 17th International Reliability Physics Symposium, Apr. 1979, pp. 197-208.

[15] L. Brookhaven National, "BNL - Gamma Radiation Source Facility," accessed on: 2020-12-15. [Online]. Available: https://www.bnl.gov/nsrl/grsf/

[16] C. La Tessa, M. Sivertz, I.-H. Chiang, D. Lowenstein, and A. Rusek, "Overview of the NASA space radiation laboratory," Life Sciences in Space Research, vol. 11, pp. 18-23, Nov. 2016.
[17] MIL-STD-883K-1019.8, "Test Standard Microcircuits," Department of Defence, Tech. Rep., 2019.

[18] M. S. Andjelković, G. S. Ristić, and A. B. Jakšić, "Using RADFET for the real-time measurement of gamma radiation dose rate," Measurement Science and Technology, vol. 26, no. 2, p. 025004, Feb. 2015.

[19] B. Abel, R. M. Thorne, and A. L. Vampola, "Solar cyclic behavior of trapped energetic electrons in Earth's inner radiation belt," Journal of Geophysical Research: Space Physics, vol. 99, no. A10, pp. 19427$19431,1994$.

[20] T. Oldham and F. McLean, "Total ionizing dose effects in MOS oxides and devices," IEEE Transactions on Nuclear Science, vol. 50, no. 3, pp. 483-499, Jun. 2003.

[21] M. O'Bryan, K. LaBel, R. Reed, R. Ladbury, J. Howard, S. Buchner, J. Barth, S. Kniffin, C. Seidleck, C. Marshal, P. Marshal, H. Kim, D. Hawkins, M. Carts, J. Forney, A. Sanders, S. Cox, C. Dunsmore, and C. Palor, "Recent radiation damage and single event effect results for candidate spacecraft electronics," Jul. 2001, pp. 82-99.

[22] M. Dominguez-Pumar, C. R. Bheesayagari, S. Gorreta, G. LopezRodriguez, and J. Pons-Nin, "Closed-Loop Compensation of Charge Trapping Induced by Ionizing Radiation in MOS Capacitors," IEEE Transactions on Industrial Electronics, vol. 65, no. 3, pp. 2518-2524, Mar. 2018. 